\title{
The NMDA antagonist MK-801 disrupts reconsolidation of a cocaine-associated memory for conditioned place preference but not for self-administration in rats
}

\author{
Travis E. Brown, Brian R. Lee, and Barbara A. Sorg ${ }^{1}$ \\ Alcohol and Drug Abuse Research Program and Program in Neuroscience, Department of Veterinary and Comparative Anatomy, \\ Pharmacology and Physiology, Washington State University, Pullman, Washington 99164-6520, USA
}

\begin{abstract}
Recent research suggests that drug-related memories are reactivated after exposure to environmental cues and may undergo reconsolidation, a process that can strengthen memories. Conversely, reconsolidation may be disrupted by certain pharmacological agents such that the drug-associated memory is weakened. Several studies have demonstrated disruption of memory reconsolidation using a drug-induced conditioned place preference (CPP) task, but no studies have explored whether cocaine-associated memories can be similarly disrupted in cocaine self-administering animals after a cocaine priming injection, which powerfully reinstates drug-seeking behavior. Here we used cocaine-induced CPP and cocaine self-administration to investigate whether the N-methyl-D-aspartate receptor antagonist (+)-5methyl-10,11-dihydro-5H-dibenzo[a,d]cyclohepten-5,10-imine maleate (MK-80I) given just prior to reactivation sessions would suppress subsequent cocaine-primed reinstatement (disruption of reconsolidation). Systemic injection of MK-801 (0.05 or $0.20 \mathrm{mg} / \mathrm{kg}$ administered intraperitoneally) in rats just prior to reactivation of the cocaine-associated memory in the CPP context attenuated subsequent cocaine-primed reinstatement, while no disruption occurred in rats that did not receive reactivation in the CPP context. However, in rats trained to self-administer cocaine, systemic administration of MK-801 just prior to either of two different types of reactivation sessions had no effect on subsequent cocaine-primed reinstatement of lever-pressing behavior. Thus, systemic administration of MK-801 disrupted the reconsolidation of a cocaine-associated memory for CPP but not for self-administration. These findings suggest that cocaine-CPP and self-administration do not use similar neurochemical processes to disrupt reconsolidation or that cocaine-associated memories in self-administering rats do not undergo reconsolidation, as assessed by lever-pressing behavior under cocaine reinstatement conditions.
\end{abstract}

The ability to disrupt previously consolidated memories in a reactivation-dependent manner is thought to be due to the disruption of a memory reconsolidation process. This disruption of reconsolidation has been observed in a wide variety of tasks and species (Nader et al. 2000b; Sara 2000; Alberini 2005; Riccio et al. 2006). Early reconsolidation experiments primarily focused on aversive learning paradigms, with an emphasis on disruption of reconsolidation as a potential treatment for posttraumatic stress disorder (Misanin et al. 1968; Nader et al. 2000a; Debiec and Ledoux 2004; Brunet et al. 2008). Only more recently have investigators demonstrated that appetitive memories also undergo reconsolidation; most, but not all (Yim et al. 2006), studies found a disruption of expression for the drug-associated memory, suggesting the potential to target the reconsolidation process as a treatment for drug addiction (Lee et al. 2005; Miller and Marshall 2005; Milekic et al. 2006; Valjent et al. 2006; Brown et al. 2007; Kelley et al. 2007; Sadler et al. 2007; Fricks-Gleason and Marshall 2008; Milton et al. 2008a,b).

Miller and Marshall (2005) showed that reconsolidation of cocaine conditioned place preference (CPP) in the rat could be disrupted by either pre- or post-treatment of a phosphorylation inhibitor of extracellular signal-regulated kinase (1/2) (ERK) in a reactivation-dependent manner. Other studies have shown that

\footnotetext{
1 Corresponding author.
}

E-mail sorg@vetmed.wsu.edu; fax (509) 335-4650.

Article is online at http://www.learnmem.org/cgi/doi/10.1101//m.1152808. protein synthesis inhibitors (Milekic et al. 2006), a matrix metalloproteinase (MMP) inhibitor (Brown et al. 2007), a $\beta$ noradrenergic receptor antagonist (Bernardi et al. 2006; Robinson and Franklin 2007a; Fricks-Gleason and Marshall 2008), and an N-methyl-D-aspartate (NMDA) receptor antagonist (Kelley et al. 2007; Sadler et al. 2007) can also disrupt the reconsolidation of drug-associated CPP memories. Studies by Lee and colleagues have shown that Zif268 antisense oligodeoxynucleotide infused into the basolateral amygdala prior to reactivation of memory for a cocaine-associated cue (the conditioned stimulus or CS) disrupts the ability of cocaine-associated cues to establish subsequent acquisition of a new instrumental response (Lee et al. 2005), and the ability of a drug-associated cue to induce relapse under a second-order schedule (Lee et al. 2006a). Thus, cocaineassociated memories appear to undergo reconsolidation in both Pavlovian and operant conditioning paradigms.

Relapse to drug-seeking or drug-taking behavior can occur after re-exposure to three types of stimuli: the drug itself, drugassociated contextual and discrete cues, and stress; and all of these may promote relapse in humans (for review, see Epstein et al. 2006). Only a few CPP studies (Valjent et al. 2006; Brown et al. 2007) and no self-administration studies to our knowledge have tested whether the drug-associated memory can be rendered susceptible to disruption by pharmacological agents such that subsequent cocaine-primed reinstatement is suppressed. This drug-primed effect is observed in humans, producing relapse (Ludwig et al. 1974; Jaffe et al. 1989), and in rats, producing 
robust reinstatement of drug-seeking behavior in both CPP and self-administration tasks (McFarland and Ettenberg 1997; McFarland and Kalivas 2001; Sanchez and Sorg 2001; Kalivas and McFarland 2003). The development of a treatment strategy that makes use of the reconsolidation process will ultimately need to be powerful enough to diminish drug-seeking behavior in the presence of sizable doses of the drug itself. Therefore, the primary goal of this study was to determine whether drug-primed reinstatement could be suppressed in rats that have the memory reactivated in the presence of a pharmacological agent in cocaine self-administering rats. Since we previously have demonstrated the ability to disrupt cocaine-primed reinstatement only in animals in which the memory was reactivated using cocaineinduced CPP, we also tested the extent to which the same parameters used to disrupt reconsolidation in a cocaine-induced CPP task would disrupt reconsolidation in a cocaine selfadministration task under conditions of drug-induced reinstatement.

To examine this question, we chose the noncompetitive NMDA receptor antagonist (+)-5-methyl-10,11-dihydro-5Hdibenzo[a,d]cyclohepten-5,10-imine maleate (MK-801). MK-801 has been shown to disrupt reconsolidation of spatial tasks (Przybyslawski and Sara 1997), fear tasks (Lee et al. 2006b), amphetamine-induced CPP (Sadler et al. 2007), cocaine-induced CPP (Kelley et al. 2007), and sucrose self-administration (Lee and Everitt 2008). Importantly, the two studies examining CPP using MK-801 did not explore whether MK-801 suppressed drugseeking behavior in a manner that was dependent on whether the memory was reactivated, leaving open the possibility that it was not a reconsolidation process that was disrupted by MK-801.

Here we demonstrate that MK-801 injected prior to cocaineprimed reinstatement of CPP disrupted subsequent cocaineprimed reinstatement of CPP, and this disruption was dependent on CPP contextual reactivation since injection of MK-801 and cocaine in the home cage did not disrupt subsequent cocaineprimed reinstatement of CPP. However, drug-seeking behavior in animals trained for cocaine self-administration was not disrupted when rats were reactivated under the same parameters that disrupted cocaine-induced CPP or when rats were given a reactivation session identical to their self-administration sessions. We thus demonstrate for the first time that memories associated with cocaine-induced CPP and cocaine self-administration are not similarly susceptible to disruption by MK-801.

\section{Results}

\section{Cocaine-primed reinstatement of CPP is attenuated by prior MK-801 treatment only in rats that had prior memory reactivation}

The results from Experiment 1 examining the influence of MK$801(0.05 \mathrm{mg} / \mathrm{kg}$ or $0.20 \mathrm{mg} / \mathrm{kg})$ on cocaine-seeking behavior in the cocaine-induced CPP task are shown in Figure 1A. The Saline controls and the high-dose MK-801 group demonstrated significant place preference on the Test Day followed by extinction (one-way ANOVA for saline $F_{(3,47)}=13.6, P<0.0001$; for $0.2 \mathrm{mg}$ / $\left.\operatorname{kg~MK-801~} F_{(3,31)}=5.6, P<0.005\right)$. For the low dose of MK-801, a two-way ANOVA revealed a significant day effect $\left(F_{(3,51)}=13.6\right.$, $P<0.0001)$, but only a trend toward a treatment $\times$ day interaction $\left(F_{(3,51)}=2.31, P<0.087\right)$. For the high dose of MK-801, there was a significant day effect $\left(F_{(3,54)}=17.8, P<0.0001\right)$ and a treatment $\times$ day interaction $\left(F_{(3,54)}=2.8, P<0.047\right)$. Post-hoc analysis indicated that on the Reinstatement Day, when no injection of MK-801 was given, the group pretreated with MK-801 had a significantly reduced reinstatement compared with controls. The difference in preference scores between controls and

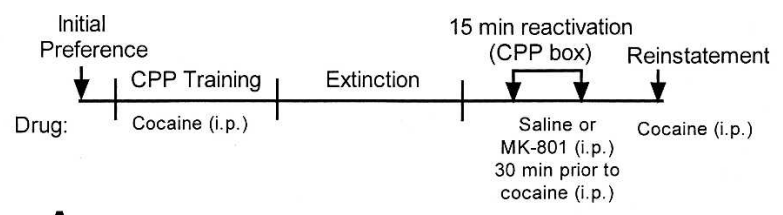

A
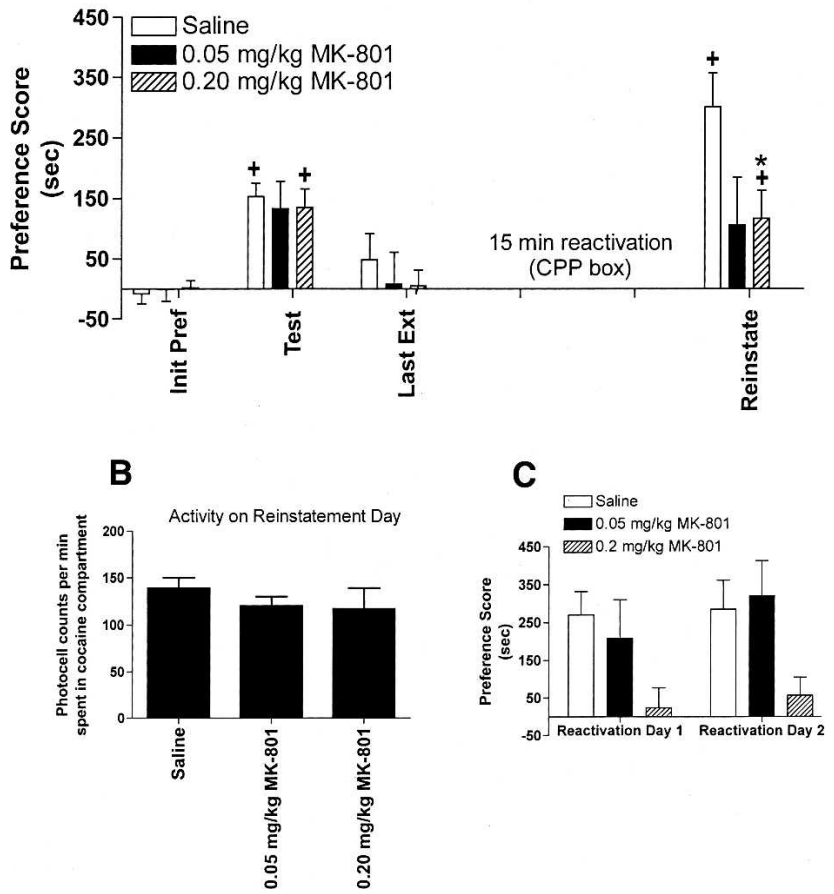

Figure 1. MK-801 prior to two reactivation sessions in the CPP chamber suppresses subsequent cocaine-primed reinstatement. Data are mean \pm SEM of preference score. Preference score $=$ day being tested minus initial preference day. (Top) The protocol for results shown in $A-C$. Init Pref = initial preference for cocaine-paired chamber prior to training; Test = test day for place preference in cocaine-paired chamber in which no cocaine was administered; Last Ext = Last Extinction Day; Reinstate $=$ cocaine-primed reinstatement using $10 \mathrm{mg} / \mathrm{kg}$ i.p. cocaine Rats were tested immediately after injection for reinstatement. Only the last extinction day is shown for clarity. $N=12$ for Saline group; $N=7$ for $0.05 \mathrm{mg} / \mathrm{kg}$ MK-801 group; $N=8$ for $0.20 \mathrm{mg} / \mathrm{kg}$ MK-801 group. ${ }^{+} P<0.05$, compared within groups to their Init Pref Day; ${ }^{*} P<0.05$, compared with saline group on the same day. $(B)$ Locomotor activity during the Reinstatement Day. Data are mean \pm SEM photocell beam breaks per minute of time spent in the cocaine-paired compartment. (C) Preference scores during the two reactivation days in the presence of saline or MK-801. Data are mean \pm SEM of preference score. The $0.20 \mathrm{mg} / \mathrm{kg}$ MK-801 dose suppressed the preference scores on both reactivation days.

MK-801-treated rats on the Reinstatement Day was not due to differences in locomotor activity (Fig. 1B), in which activity in the cocaine-paired compartment was assessed (photocell beam breaks per minute of time spent in the cocaine-paired compartment). Figure $1 \mathrm{C}$ shows the behavior from CPP during the two reactivation days. A two-way ANOVA conducted for each dose of MK-801 versus Saline with a repeated measure over day revealed that the administration of $0.05 \mathrm{mg} / \mathrm{kg}$ MK-801 $30 \mathrm{~min}$ prior to Reactivation Day 1 or Reactivation Day 2 had no effect on the expression of CPP compared with controls. For the 0.2 $\mathrm{mg} / \mathrm{kg}$ MK-801 dose, there was a significant treatment effect $\left(F_{(1,18)}=7.8, P>0.012\right)$, such that MK-801 given prior to reactivation attenuated the preference scores on Reactivation Days 1 and 2. Locomotor activity, again expressed as activity in the cocaine-paired compartment per minute of time spent in this 
compartment, was not different for either dose of MK-801, as determined by a Student's $t$-test comparing separately the doses of MK-801 with saline on Reactivation Day 2 (Saline $=146 \pm 8$; $0.05 \mathrm{mg} / \mathrm{kg}$ MK-801 = $146 \pm 22 ; 0.2 \mathrm{mg} / \mathrm{kg}$ MK-801 = $114 \pm 22$; Reactivation Day 1 locomotor data was not available).

It is possible that the effects of MK-801 were not due to disruption of reconsolidation but to other nonspecific carry-over effects of the drug on the Reinstatement Day. To test for this possibility, Experiment 2 examined the effect of administering MK-801 $(0.20 \mathrm{mg} / \mathrm{kg})$ in the home cage instead of in the CPP apparatus. The $0.20 \mathrm{mg} / \mathrm{kg}$ dose was chosen because it was expected that any carry-over effects would be most pronounced in this treatment group. Our previous work indicated that memory disruption was contingent upon reactivation by both cocaine and the CPP apparatus; that is, the cocaine priming injection needed to be given in the CPP apparatus for it to effectively reactivate the cocaine-associated memory and be subsequently disrupted (Brown et al. 2007). Consequently, this group also served as a nonreactivation control. For the two Reactivation Days, we administered saline or MK-801 followed by cocaine in the home cage. The results of Experiment 2 are shown in Figure 2. Figure $2 \mathrm{~A}$ shows that both Saline control and the high-dose MK-801 groups demonstrated significant place preference on the

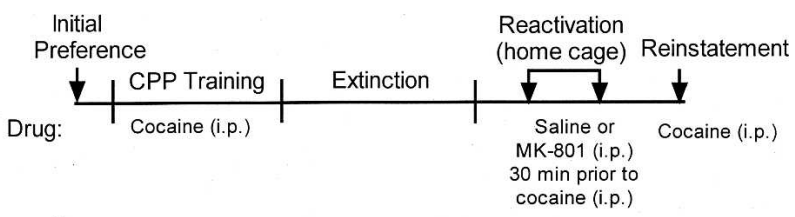

A

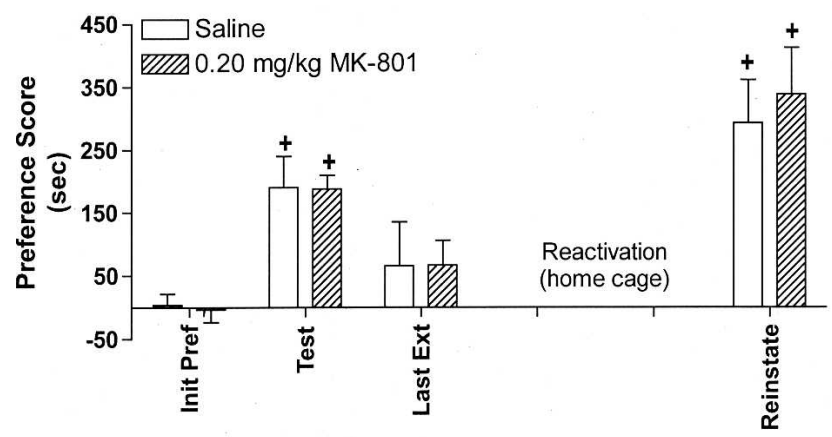

B

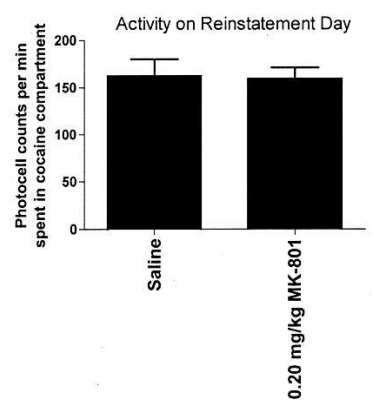

Figure 2. MK-801 prior to two reactivation sessions in the home cage does not suppress subsequent cocaine-primed reinstatement. Data are mean \pm SEM of preference score. (Top) The protocol for results shown in $A$ and $B$. See Figure 1 legend for full explanation of terminology. Drugs were administered in the home cage on Reactivation Days 1 and 2 instead of in the CPP apparatus. $N=7$ for Saline group; $N=8$ for 0.20 $\mathrm{mg} / \mathrm{kg}$ MK-801 group. ${ }^{+} P<0.05$, compared within groups to their Init Pref Day. (B) Locomotor activity during the Reinstatement Day. Data are mean \pm SEM photocell beam breaks per minute of time spent in the cocaine-paired compartment.
Test Day followed by extinction and significant reinstatement (one-way ANOVA for saline $F_{(3,27)}=6.9, P<0.003$; for $0.2 \mathrm{mg} / \mathrm{kg}$ MK-801 $\left.F_{(3,31)}=13.1, P<0.0001\right)$. A two-way ANOVA revealed a significant day effect $\left(F_{(3,39)}=19.5, P<0.0001\right)$ but no other differences, indicating that the combination of $0.2 \mathrm{mg} / \mathrm{kg} \mathrm{MK}-801$ with cocaine given on Reactivation Days 1 and 2 in the home cage did not impair subsequent cocaine-primed reinstatement in the CPP apparatus on the Reinstatement Day. We also did not observe any effects of this prior dose of MK-801 on locomotor activity in the cocaine-paired compartment on the Reinstatement Day (Fig. 2B).

\section{Cocaine-primed reinstatement of lever-pressing behavior is not attenuated by prior $\mathrm{MK}-801$ treatment in rats trained for cocaine self-administration}

In Experiment 3 we tested whether the same reactivation parameters applied to the CPP procedure would disrupt reconsolidation when rats were instead trained in a cocaine self-administration task. The results are shown in Figure 3A. One-way ANOVAs for each treatment group showed that all three groups demonstrated extinction followed by reinstatement (for Saline day $F_{(4,84)}=84.1, P<0.0001$; for $0.05 \mathrm{mg} / \mathrm{kg}$ MK-801 $F_{(4,49)}=15.1$, $P<0.0001$; for $\left.0.2 \mathrm{mg} / \mathrm{kg} \mathrm{MK}-801 F_{(4,79)}=50.1, P<0.0001\right)$. Analysis of the comparison between Saline controls and the 0.05 $\mathrm{mg} / \mathrm{kg}$ MK-801 group by a two-way ANOVA revealed a significant day effect $\left(F_{(4,100)}=65.9, P<0.0001\right)$, and for $0.2 \mathrm{mg} / \mathrm{kg} \mathrm{MK}-801$, a significant day effect $\left(F_{(4,124)}=127, P<0.0001\right)$, but no other significant effects. Thus, prior MK-801 treatment during reactivation did not impact subsequent cocaine-induced reinstatement compared with controls, indicating that reconsolidation was not disrupted by MK-801 treatment. Analysis of the number of rewards during the last three Maintenance Days revealed that $0.05 \mathrm{mg} / \mathrm{kg}$ MK-801-treated rats were significantly higher than Saline controls on the middle day (Maint 2), as determined by a two-way ANOVA followed by a least significant difference test (Maintenance Day 1: Saline $=21.3 \pm 1.2 ; 0.05$ $\mathrm{mg} / \mathrm{kg}$ MK-801 $=22.8 \pm 2.3 ;$ Maintenance Day 2: Saline $=21.2 \pm 1.1 ; 0.05 \mathrm{mg} / \mathrm{kg} \mathrm{MK}-801=23.7 \pm 2.2$; Maintenance Day 3: Saline $=23.1 \pm 1.3 ; 0.05 \mathrm{mg} / \mathrm{kg}$ MK-801 $=22.3 \pm 1.5$; $\left.F_{(2,50)}=3.5, P<0.039\right)$. For the $0.2 \mathrm{mg} / \mathrm{kg} \mathrm{MK}-801$ group, the number of rewards was not different from Saline controls (both groups ranged between 21 and 23 rewards across days). Figure 3B shows the number of inactive lever presses during maintenance, extinction, and reinstatement. For the Saline and $0.2 \mathrm{mg} / \mathrm{kg}$ MK801 groups, there were no significant differences across day, as determined by a one-way ANOVA for each group. For the 0.05 $\mathrm{mg} / \mathrm{kg}$ MK-801 group, a one-way ANOVA revealed a significant day effect $\left.F_{(4,49)}=6.2, P<0.0007\right)$, with rats demonstrating a decrease in lever presses on the last extinction day compared with the reinstatement day. A two-way ANOVA comparing Saline controls with each dose of MK-801 revealed only a significant day effect for the $0.05 \mathrm{mg} / \mathrm{kg}$ MK-801 versus Saline comparison $\left.F_{(4,100)}=4.4, P<0.003\right)$. Figure $3 \mathrm{C}$ shows the number of active lever presses during the 15-min time period on Reactivation Days 1 and 2. While there was a strong trend for an elevation in lever presses in the $0.05 \mathrm{mg} / \mathrm{kg}$ MK-801 group (treatment $F_{(1,25)}=4.0$, $P<0.056)$, there were no effects of $0.2 \mathrm{mg} / \mathrm{kg}$ MK- 801 on active lever pressing. This strong trend toward enhanced lever pressing in the $0.05 \mathrm{mg} / \mathrm{kg}$ MK-801 group is likely not due to enhanced cocaine-induced locomotion, at least as assessed in the CPP test from a different group of rats shown in Figure 1, since locomotor activity after cocaine injection was identical to Saline controls (see text above describing Fig. 1C). There were no significant differences in the number of inactive lever presses between saline and MK-801-treated animals during the two 15-min Reactivation 


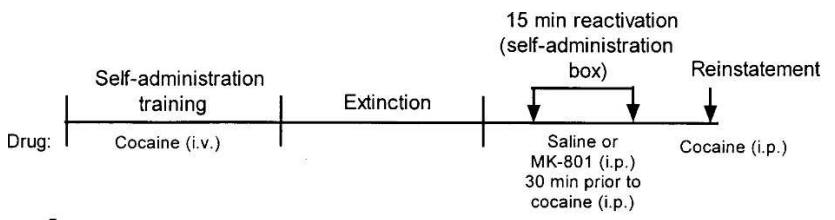

A
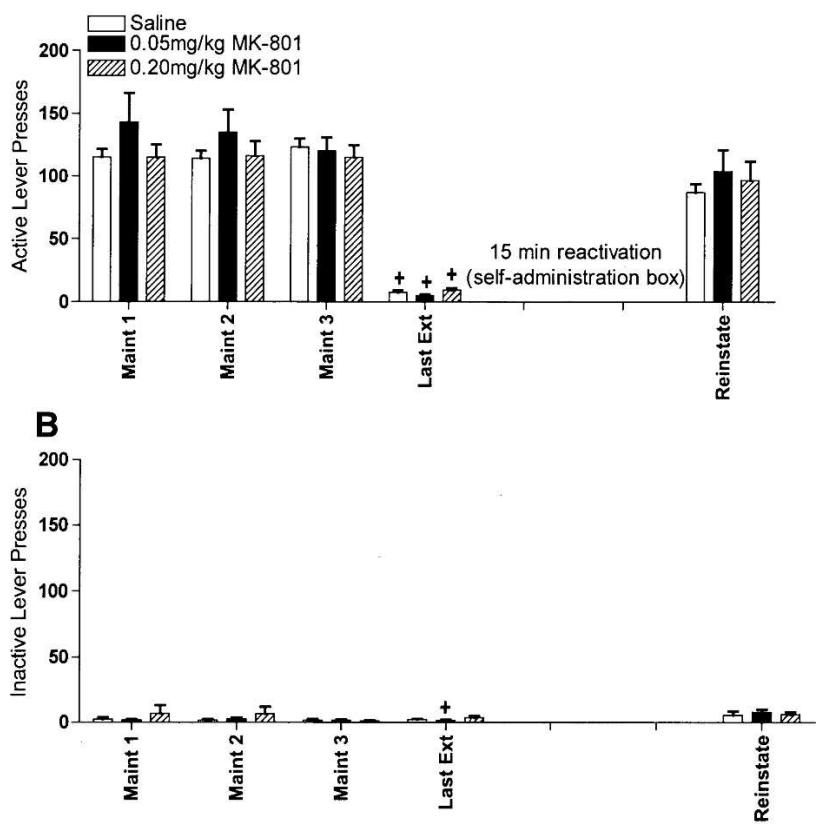

C

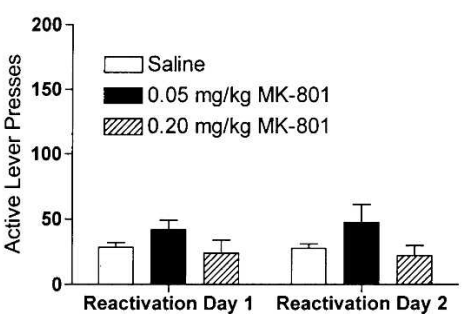

Figure 3. MK-801 prior to two 15-min noncontingent cocaine presentations in the self-administration chamber does not suppress subsequent cocaine-primed reinstatement. Data are mean \pm SEM of active lever presses $(A)$ and inactive lever presses $(B)$. (Top) The protocol for results shown in $A-C$. For clarity, only the last $3 \mathrm{~d}$ of the self-administration maintenance phase are shown (Maint 1-3). Maint = Active lever presses during maintenance training; Last Ext = Last Extinction Day; Reinstate $=$ cocaine-primed reinstatement using $10 \mathrm{mg} / \mathrm{kg}$ i.p. cocaine. Rats were tested in a 2-h session immediately after injection for reinstatement. Only the last extinction day is shown for clarity. $(A) N=17$ for Saline group; $N=10$ for $0.05 \mathrm{mg} / \mathrm{kg} \mathrm{MK}-801$ group; $N=16$ for 0.20 $\mathrm{mg} / \mathrm{kg} \mathrm{MK-801} \mathrm{group.} \mathrm{(B)} N=15$ for the $0.20 \mathrm{mg} / \mathrm{kg}$ MK-801 group because of one rat that pressed over 2300 times on the inactive lever during the third maintenance day and was removed from the analysis and the figure. ${ }^{+} P<0.05$, compared within groups between Last Extinction Day and the Reinstatement Day (for clarity, only significant differences between these two days are indicated). (C) Active lever presses during the two reactivation days in the presence of saline or MK-801. Data are mean \pm SEM of active lever presses.

Days, as determined by two-way ANOVAs separately comparing Saline controls with each dose of MK-801 (Reactivation Day 1: Saline $=0.9 \pm 0.4 ; 0.05 \mathrm{mg} / \mathrm{kg}$ MK-801 $=2.4 \pm 1.1 ; 0.20 \mathrm{mg} / \mathrm{kg}$ MK-801 = $6.2 \pm 4.1 ;$ Reactivation Day 2: Saline $=1.2 \pm 0.4 ; 0.05$ $\mathrm{mg} / \mathrm{kg}$ MK-801 = $1.9 \pm 1.3 ; 0.20 \mathrm{mg} / \mathrm{kg} \mathrm{MK}-801=1.4 \pm 0.8$ ). The number of days to acquisition or extinction was not different among any of the three treatment groups as determined by one-way ANOVAs comparing separately Saline controls with each dose of MK-801 (Days to acquisition: Saline $=14.0 \pm 0.5$; $0.05 \mathrm{mg} / \mathrm{kg} \mathrm{MK}-801=15.3 \pm 0.7 ; 0.2 \mathrm{mg} / \mathrm{kg} \mathrm{MK}-801=$ $15.4 \pm 1.0 ;$ Days to extinction: Saline $=8.4 \pm 0.9 ; 0.05 \mathrm{mg} / \mathrm{kg}$ MK-801 = $8.8 \pm 1.0 ; 0.2 \mathrm{mg} / \mathrm{kg}$ MK-801 = $7.9 \pm 0.6$ ).

Because we did not observe an effect of MK-801 on drugseeking behavior after the 15-min reactivation sessions, we reasoned that reactivation sessions with noncontingent cocaine injections might not sufficiently reactivate the memory of selfadministration because the investigator-administered cocaine priming injection may be perceived as a novel experience by self-administering rats. Therefore, in Experiment 4 we tested whether reactivation sessions identical to the daily maintenancephase self-administration session would more effectively reactivate the cocaine-associated memory, rendering this memory more susceptible to disruption by MK-801 administration. The results from Experiment 4 are shown in Figure 4.

One-way ANOVAs for both treatment groups showed that both groups demonstrated extinction followed by reinstatement (Fig. 4A; for Saline, day $F_{(4,39)}=63.1, P<0.0001$; for $0.2 \mathrm{mg} / \mathrm{kg}$ MK-801, $\left.F_{(4,39)}=20.0, P<0.0001\right)$. A two-way ANOVA comparing Saline controls with the MK-801 group revealed only an effect of day $\left(F_{(4,56)}=59.8, P<0.0001\right)$ but no other effects. These results indicate that prior MK-801 given during the reactivation sessions did not impact subsequent cocaine-induced reinstatement, indicating that reconsolidation was not disrupted by MK801 treatment, similar to what we found in Experiment 3 using the 15-min noncontingent cocaine during the reactivation sessions. The number of rewards during the last three Maintenance Days was not different between controls and rats to be treated with MK-801 (both groups had between 22 and 23 rewards). Figure $4 \mathrm{~B}$ shows the number of inactive lever presses during maintenance, extinction, and reinstatement. Separate one-way ANOVAs for each of the two groups revealed a day effect for Saline controls $\left(F_{(4,39)}=5.1, P<0.003\right)$, but post-hoc analysis did not reveal differences between the last extinction day and the reinstatement day. There were no significant effects in the 0.2 $\mathrm{mg} / \mathrm{kg}$ MK-801 group. A two-way ANOVA comparing Saline controls and the MK-801 group revealed only a significant day effect $\left(F_{(4,56)}=3.4, P<0.014\right)$. Figure $4 \mathrm{C}$ shows the number of active lever presses during the 2-h reactivation sessions on Reactivation Days 1 and 2. A two-way ANOVA comparing Saline controls and MK-801-treated rats revealed a significant treatment effect $\left(F_{(1,14)}=11.6, P<0.004\right)$, with the MK-801 rats showing a decreased number of lever presses during these reactivation sessions. There were no differences between groups in the number of inactive lever presses during Reactivation Days 1 and 2 (all values for inactive lever presses were less than 1). The number of days to acquisition was not different between the two treatment groups (Saline controls $=12.0 \pm 0.4 ; \mathrm{MK}-801=12.6 \pm 0.7$ ). The number of days to reach extinction was significantly less in the MK-801 group (Saline control $=8.6 \pm 0.8$; MK-801 = $6.5 \pm 0.5$; $\left.F_{(1,15)}=3.4, P<0.038\right)$. Overall, the findings from Experiments 3 and 4 demonstrate that whether 15 -min, noncontingent cocaine priming injections or 2-h cocaine self-administration sessions were given for the two Reactivation Days, MK-801 did not attenuate subsequent cocaine-primed reinstatement, suggesting that reconsolidation of the cocaine-associated memory was not disrupted by this agent in rats trained to self-administer cocaine.

\section{Discussion}

The main findings from this study are: (1) MK-801 impairs the reconsolidation of the cocaine-associated context in a CPP task, even while animals are under the influence of cocaine during reinstatement testing; (2) disruption of reconsolidation by 


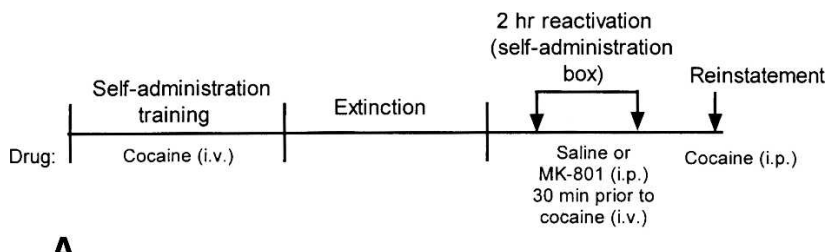

A

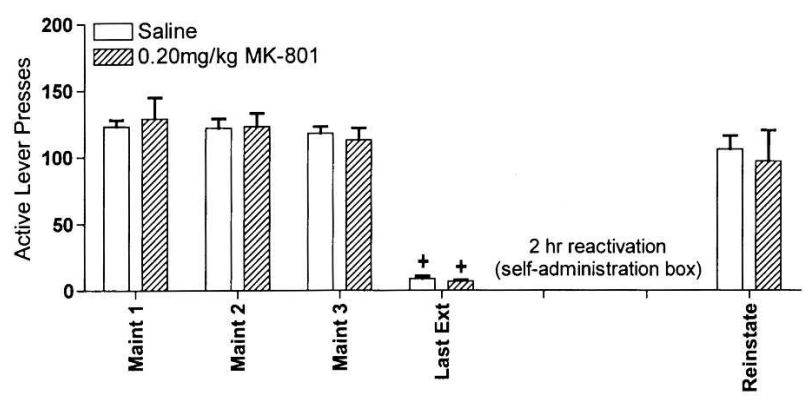

B

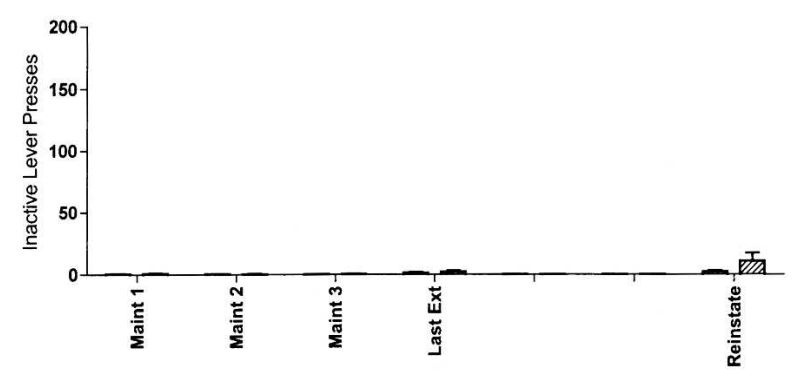

C

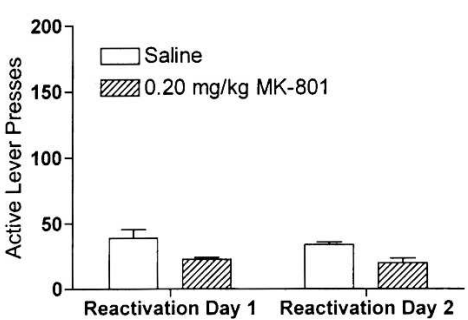

Figure 4. MK-801 prior to two 2-h cocaine self-administration reactivation sessions in the self-administration chamber does not suppress subsequent cocaine-primed reinstatement. Data are mean \pm SEM of active lever presses $(A)$ and inactive lever presses $(B)$. (Top) The protocol for results shown in $A-C$. See Figure 3 legend for full explanation of terminology. Rats were tested in a 2 -h session immediately after injection for reinstatement. $(A, B) N=8$ for each group. ${ }^{+} P<0.05$, compared within groups between Last Extinction Day and the Reinstatement Day (for clarity, only significant differences between these two days are indicated). (C) Active lever presses during the two reactivation days in the presence of saline or MK-801. Data are mean \pm SEM of active lever presses. MK-801 suppressed lever pressing on both reactivation days.

MK-801 appears to require reactivation of the cocaine-associated memory in the CPP context; and (3) MK-801 does not disrupt the reconsolidation of a cocaine-associated memory in rats trained for cocaine self-administration when this memory is reactivated under the same parameters that disrupt cocaine-CPP or when the memory is reactivated with standard 2-h cocaine selfadministration sessions.

Our findings describing the ability of MK-801 to disrupt the reconsolidation of a drug-CPP task are in accordance with two recent studies (Kelley et al. 2007; Sadler et al. 2007). However, neither of these studies included nonreactivated groups, leaving open the possibility for alternative interpretations to explain the ability of MK-801 to disrupt CPP. Here we show that the suppression of place preference behavior after prior MK-801 treatment occurred only when the memory was reactivated; that is, we saw suppression only when animals were exposed to the CPP apparatus simultaneously with MK-801. Thus, our studies support the idea that reconsolidation has been disrupted by MK-801 given during reactivation sessions.

We have previously shown that disruption of reconsolidation required reactivation sessions in which rats were simultaneously exposed to cocaine and the CPP context (Brown et al. 2007). Consistent with this finding, in this study we found that cocaine + MK-801 treatments in the home cage did not disrupt reconsolidation. However, the reports from different CPP studies are mixed with regard to the requirements for stimuli present during reactivation. For example, Miller and Marshall (2005) showed that inhibition of the ERK pathway could disrupt reconsolidation of a cocaine-CPP task when reactivated in the CPP context alone, while Valjent et al. (2006) showed that inhibition of the ERK pathway disrupted reconsolidation of cocaine-CPP task but required reactivation using both the drug and the CPP context. Similarly, Milekic et al. (2006) demonstrated that reconsolidation of a morphine-associated memory was disrupted by protein synthesis inhibitors only when rats received a reactivation session with morphine while located inside the CPP chambers. Further, not all studies have demonstrated disruption of drug-associated place preference using the retrieval approach; Yim et al. (2006) did not observe impaired morphine-associated place preference when a protein synthesis inhibitor was delivered into the basolateral amygdala during reactivation. Some of the variability observed between the above studies may be due to differences in the route of administration of the disrupting agent (central vs. peripheral) or the species examined (rat vs. mouse). Even studies that have employed similar reactivation parameters have shown variable results when attempting to disrupt reconsolidation of morphine-CPP by anisomycin (Milekic et al. 2006; Robinson and Franklin 2007b).

Alternative explanations are considered for the disruption of reconsolidation in our CPP studies. The first possibility is that MK-801 is causing a general memory loss. However, this appears unlikely because rats treated with MK-801 in the home cage demonstrated normal cocaine-primed reinstatement compared with controls (Fig. 2), and, additionally, MK-801 did not disrupt lever pressing in the self-administration task (Figs. 3 and 4). Another possibility is that MK-801 accelerates extinction. This also appears unlikely because extinction is new learning that is defined by four different phenomena (Bouton 2004), one of which is the ability of the unconditioned stimulus to reinstate the conditioned behavioral response. In this study, reinstatement was significantly suppressed compared with controls when the high dose of MK-801 was used. Also, Sadler et al. (2007) showed that repeated administration of MK-801 prior to 10 separate extinction trials did not facilitate extinction, while another NMDA antagonist, 3(-2 carboxipiperazin-4-yl)propyl-1-phosphonic acid, has been shown to disrupt, rather than accelerate, extinction consolidation in a cocaine-CPP task (Kelamangalath et al. 2007). It is possible that MK-801 produces a negative physiological state that the animal associates with the cocaine-paired context; such an aversive effect has been described using the protein synthesis inhibitor anisomycin to disrupt both CPP (Robinson and Franklin 2007b) and an appetitive instrumental task (Hernandez and Kelley 2004). However, rats were not explicitly paired with MK801 in the cocaine compartment on the two reactivation days; they were allowed to freely explore all three compartments. Thus, an aversive response to cocaine does not seem to be a likely explanation. Also, if we did produce aversion, we might expect to 
observe reduced active lever pressing during reinstatement in the self-administration task, which we did not.

Pharmacological manipulations that disrupt CPP generally disrupt self-administration (de Wit and Stewart 1981; Stewart and Grupp 1989; Mueller and Stewart 2000; Parker and McDonald 2000; Kalivas and McFarland 2003). However, Bardo et al. (1999) showed that the amount of time spent in the amphetamine-paired compartment in a CPP task did not predict the amount of lever pressing a rat did in a self-administration task, even though the dopamine antagonist, SCH-23390, was able to block drug-seeking behavior in both a CPP task and a selfadministration task. Leri et al. (2006) showed that methadone treatment blocked cocaine-CPP but not cocaine self-administration. Thus, there are recognized differences between these two types of tasks.

The ability to disrupt reconsolidation appears to hinge upon the reactivation parameters used. In general, recent memories have been shown to be disrupted more easily than older (aged) memories (Litvin and Anokhin 2000; Milekic and Alberini 2002; Suzuki et al. 2004). However, others have described disruptive effects of protein synthesis inhibitors or other drugs in older or stronger memories (Nader et al. 2000a; Debiec et al. 2002; Lee et al. 2006a). Suzuki et al. (2004) and Frankland et al. (2006) have demonstrated that older or stronger memories were disrupted by anisomycin if longer reactivation times (i.e., exposure time to the stimulus) were applied. It is difficult to envision that the 2-h reactivation sessions used in our self-administration studies were not long enough to reactivate the memory, but it is possible that additional reactivation sessions are required for MK-801 or any agent to sufficiently disrupt reconsolidation of the cocaineassociated memory. Self-administration likely produces a "stronger" memory than does CPP training because of the amount of cocaine and/or the number of pairings of cocaine with the environment in rats undergoing self-administration compared with CPP. Rats given CPP training received an average of $\sim 17 \mathrm{mg}$ of i.p. cocaine, with 4 cocaine + context pairings, while rats given selfadministration training received $\sim 96 \mathrm{mg}$ of i.v. cocaine and an average of 366 cocaine + context + cue light pairings. A study by Lee et al. (2006a) is the only one we are aware of that has examined the ability of a pharmacological agent to disrupt the reconsolidation of cocaine-seeking behavior in a self-administration task in the presence of cocaine. In that study, they used a secondorder schedule of cocaine reinforcement and demonstrated that rats given Zif268 antisense oligodeoxynucleotide into the basolateral amygdala during a reactivation session suppressed subsequent cocaine-seeking behavior during the maintenance phase of self-administration. The differences between the findings of Lee et al. (2006a) and ours may be in the pharmacological agent used, the different schedule of reinforcement, the reactivation parameters (30 noncontingent presentations of the CS [light] during a 30-min session in the Lee et al. study and no cocaine present), the fact that Lee et al. (2006a) did not conduct extinction sessions as in our study, or the differences in cocaine history (rats in the Lee et al. study had an average of 210 pairings of cocaine). Finally, another difference that may be key to the discrepant findings is the amount of cocaine present on the day of testing: Lee et al. (2006a) used a $0.25 \mathrm{mg} /$ infusion (i.v.), given every $15 \mathrm{~min}$ after the tenth active lever press, versus a $10 \mathrm{mg} / \mathrm{kg}$ (i.p.) bolus injection given immediately before the start of the session in this study. The dose of cocaine used for reinstatement in our study may have sufficiently overcome any MK-801induced weakening of the cocaine-associated memory.

Several alternative explanations for the different effects of MK-801 on CPP versus self-administration behavior are considered. One possibility is that the reactivation and/or reconsolidation processes involved in these two types of tasks do not use the same neurochemical processes. For example, NMDA receptors may be required for the reconsolidation of a CPP task but not for the self-administration task. A second possibility is that in rats trained for self-administration, NMDA antagonism could impair the ability to transform a memory to a labile state to render it capable of being disrupted (Ben Mamou et al. 2006). A third possibility is that in the self-administration studies, we may have reactivated the instrumental extinction memory rather than the original drug memory during the two reactivation days, and therefore MK-801 could have impaired extinction. Supporting this idea is the significant decrease in the number of active lever presses in MK-801-treated rats on the reactivation days from Experiment 4 (Fig. 4C); thus, some extinction of active lever pressing may have occurred. However, three lines of reasoning do not support the idea that MK-801 blocked extinction of instrumental memory. First, animals were given a cocaine injection (Experiment 3) or allowed to press for cocaine (Experiment 4), and so extinction is not expected to occur in the presence of the drug. Second, none of the treatment groups demonstrated extinction between the two reactivation days. Third, in Experiment 3, we did not observe a decrease in active lever-pressing behavior in MK-801-treated rats as we did in Experiment 4 during the reactivation days, but the results on the final test for reinstatement were similar to those from Experiment 4. Extending the line of reasoning that the presence of cocaine is less likely to favor extinction behavior, the absence of cocaine during reactivation may favor a competing extinction memory to be reactivated or may prevent full reactivation of the original cocaine-associated memory. This appears to be the case for CPP studies, in which Valjent et al. (2006), Milekic et al. (2006), and Brown et al. (2007) have demonstrated that disruption of reconsolidation requires memory reactivation in the presence of both the drug and CPP chamber. A fourth possibility is that instrumental responses do not undergo reconsolidation, because these responses are welllearned and perhaps not subject to disruption. This notion was suggested by Hernandez and Kelley (2004). Further studies by Lee et al. (2006a) and Milton et al. (2008b) would suggest that amnestic agents given during reactivation do not modify the memory for the lever-reward association. In their studies, memory reactivation using the cocaine-associated cue light in the presence of an amnestic agent reduced the ability of the cue to influence the instrumental response, resulting in decreased lever-pressing behavior. In the same way that the reactivation session with the cue suppressed the ability of that cue to later activate lever-pressing behavior, we reasoned that reactivation of the cocaine + cue might disrupt the ability of interoceptive cocaine cues and/or the light cue to enhance lever-pressing behavior in the present study. The fact that we did not find disruption of lever-pressing behavior may indicate that rats trained to selfadminister cocaine do not undergo any appreciable disruption of reconsolidation when higher doses of cocaine are present at the time of reactivation and/or reinstatement.

The higher dose of MK-801 during the reactivation sessions attenuated both CPP and self-administration, but the number of active lever presses was reduced only under conditions where animals were pressing for cocaine (Experiment 4), and not under conditions in which rats received the noncontingent cocaine priming injection and were pressing for only the cocaine cues (Experiment 3). Since MK-801 alone produces reinstatement (De Vries et al. 1998; Edwards et al. 2007) and has rewarding properties as indexed by CPP behavior (Steinpreis et al. 1995; Panos et al. 1999), the reinforcing effects of MK-801 may have been additive with the effects of cocaine, and animals therefore may have reduced their cocaine-seeking behavior in the presence of MK801. Alternatively, MK-801 suppresses acute cocaine responses (Pierce et al. 1997) for reasons that are not yet clear, and this may 
also have explained the suppressed CPP and lever-pressing behavior during reactivation. Regardless of the mechanism for suppression on the reactivation days by this higher dose of MK-801, it does not appear to explain the differences in drug-seeking behavior between CPP and self-administration on the Reinstatement Day when no MK-801 was present, since animals showed lower preference for the cocaine-paired side in the CPP task on this day but were not affected in the self-administration task. The findings also suggest that although behavioral expression of CPP was impaired on the reactivation days by the higher dose of MK-801, the memory for cocaine-associated cues still appears to have been reactivated (but not expressed) because CPP was suppressed on the Reinstatement Day only in animals that were given the reactivation sessions. Evidence that memories can still undergo reconsolidation in the absence of expression of memory at the time of reactivation is supported by fear conditioning studies in which the AMPA antagonist 6-cyano-7-nitroquinoxalone2,3-dione prevented the expression of fear but not the ability of anisomycin to disrupt reconsolidation of the fear-associated CS memory (Ben Mamou et al. 2006).

In summary, our work shows for the first time that the same reactivation parameters and pharmacological agent (MK-801) that disrupted the reconsolidation of a cocaine-associated memory for a CPP task did not disrupt reconsolidation of the memory for a self-administration task. Further, reactivation parameters that mimicked the self-administration procedure itself, and therefore should have promoted robust retrieval of the cocaine-associated memory, also failed to render this memory labile for disruption by MK-801. The possibility of diminishing persistent and unwanted memories by disrupting the reconsolidation process opens exciting new frontiers for developing treatments for pathological disorders, including drug abuse. However, the complexity of memory storage and subsequent memory retrieval that ultimately may lead to memory recoding has only begun to be elucidated and therefore requires further systematic investigation with regard to the timing and the specific parameters used for reactivation.

\section{Materials and Methods}

\section{Subjects}

Male Sprague-Dawley and Long-Evans Hooded rats weighing 280-350 g at the start of the experiment were housed in a temperature- and humidity-controlled colony room with a 12-h light/dark cycle (lights on at 6:00 a.m.). Sprague-Dawley rats were used for all CPP studies, and our initial self-administration studies used Long-Evans rats because of their higher general activity levels and thus higher initial lever pressing during acquisition of the self-administration task. However, to ensure that there were no strain differences in the effects of MK-801 on selfadministration behavior, we also used Sprague-Dawley rats to test the effects of the highest dose of MK-801 compared with Saline vehicle in this strain. No significant differences were found for the effects of MK-801, so the data from both strains were pooled. Animals undergoing self-administration were housed in a 12-h reverse light/dark cycle (lights on at 6:00 p.m.). Experiments were conducted according to the National Institutes of Health Guide for the Care and Use of Laboratory Animals, and experimental protocols were approved by the University Animal Care and Use Committee. Animals were housed two per cage for the CPP studies and individually for the self-administration studies. Food and water were provided ad libitum except for when animals were engaged in experiments.

\section{Drug administration}

Cocaine hydrochloride was obtained from the Sigma-Aldrich Company and dissolved as the weight of the salt to a final concentration of $12 \mathrm{mg} / \mathrm{kg}$ (CPP training dose), $0.75 \mathrm{mg} / \mathrm{kg}$ (self- administration training dose), or $10 \mathrm{mg} / \mathrm{kg}$ (reactivation and reinstatement doses, CPP and self-administration sessions).

(+)-MK-801 hydrogen maleate was obtained from the SigmaAldrich Company and dissolved in sterile saline for i.p. injection $(1 \mathrm{~mL} / \mathrm{kg})$. The doses chosen were 0.05 and $0.20 \mathrm{mg} / \mathrm{kg}$, based on previous work by Przybyslawski and Sara (1997).

\section{Surgery}

Self-administration surgery was conducted according to a modification of McFarland and Kalivas (2001). Rats were anesthetized with zyket (ketamine $87 \mathrm{mg} / \mathrm{kg}$ + xylazine $13 \mathrm{mg} / \mathrm{kg}$ ) given intramuscularly prior to implanting a chronic indwelling i.v. catheter. The catheter was surgically implanted into the right jugular vein, and the distal end was led subcutaneously to the back between the scapulas. Catheters were constructed from Silastic tubing $(9 \mathrm{~cm}$; inner diameter 0.025 in, outer diameter $0.047 \mathrm{in})$ connected to a back-mount cannula pedestal, a bent 22-gauge metal cannula encased within a plastic screw connector attached to a polyester mesh (Plastics One). A small ball of silicone sealant was placed $\sim 2.8 \mathrm{~cm}$ from the end of the catheter. The right jugular vein was isolated, the most anterior portion of the vein was tied shut, and a small incision was made. The distal end of the catheter was inserted into the vein until the silicone ball was flush with the vein. The vein was secured by tying suture thread on both sides of the silicone ball; additionally, the thread on both sides was tied together. Immediately after surgery, the catheter was injected with $0.1 \mathrm{~mL}$ of locking solution: heparin $(500$ $\mathrm{U} / \mathrm{mL})$, gentamicin $(5 \mathrm{mg} / \mathrm{mL})$, and glycerol $(60 \%)$ in sterile saline. Incisions were sutured, and the animal was given 5-7 d to recover. After surgery, the catheter was flushed daily with $0.1 \mathrm{~mL}$ of heparin $(10 \mathrm{U} / \mathrm{mL})$ and gentamicin antibiotic $(5 \mathrm{mg} / \mathrm{mL})$ in sterile saline to help protect against infection and catheter occlusion.

\section{Behavioral procedures}

\section{CPP}

All CPP studies were conducted during the same time of day. The proposed studies employed a three-compartment CPP apparatus (Med Associates, Inc.) as previously described (Brown et al. 2007). Briefly, the procedure consisted of a preconditioning preference test, training for $8 \mathrm{~d}$ ( 4 saline pairings alternating with 4 cocaine pairings), testing for CPP acquisition followed by extinction sessions, and cocaine-primed reinstatement with a $10 \mathrm{mg} / \mathrm{kg}$, i.p. dose of cocaine (Brown et al. 2007). Except for the training days, rats had access to all three compartments of the CPP apparatus.

In Experiment 1, we tested whether MK-801 would impair reconsolidation of the memory for the cocaine-associated context during reinstatement testing. Animals underwent preconditioning, conditioning, testing, and extinction as described above, and on Reactivation Day 1, rats received saline or MK-801 (0.05 $\mathrm{mg} / \mathrm{kg}$ or $0.20 \mathrm{mg} / \mathrm{kg}$, i.p.) $30 \mathrm{~min}$ prior to a cocaine injection (10 $\mathrm{mg} / \mathrm{kg}$, i.p.) and placed immediately into the central compartment of the CPP box (Reactivation Day 1). Rats were allowed to explore all three compartments. The next day, the procedure from Reactivation Day 1 was repeated (Reactivation Day 2). This procedure was given for $2 \mathrm{~d}$ because our previous studies using a different pharmacological agent (Brown et al. 2007) indicated that one day of memory reactivation was not sufficient to disrupt subsequent cocaine-primed reinstatement. The following day, animals were tested for cocaine-primed reinstatement without any prior injection of either saline or MK-801 before being placed into the CPP box (Reinstatement Day). Rats were allowed to explore all three compartments.

Experiment 2 was identical to Experiment 1 with the exception of the cage location where MK-801 and cocaine injection took place on Reactivation Days 1 and 2. In Experiment 2, animals were given saline or MK-801 followed by cocaine $30 \mathrm{~min}$ later in the home cage instead of in the CPP apparatus for the two days of "reactivation." This was done to determine whether reactivation of the memory for the cocaine-associated context by cocaine in the CPP context was necessary for the ability of 
MK-801 to disrupt reconsolidation. Animals underwent preconditioning, conditioning, testing, and extinction as described above but animals were injected with saline or MK-801 $(0.20$ $\mathrm{mg} / \mathrm{kg}$, i.p.) $30 \mathrm{~min}$ prior to a cocaine injection $(10 \mathrm{mg} / \mathrm{kg}$, i.p.) in the home cage. Animals remained in the home cages, and the next day, the procedure from the first day of reactivation was repeated. The following day, animals were tested for cocaineprimed reinstatement in their CPP box without any prior microinjection of saline or MK-801, exactly as described for the Reinstatement Day in Experiment 1 above.

\section{Self-administration}

\section{Apparatus}

Experiments were conducted in operant-conditioning chambers enclosed within sound-attenuating cabinets (Med Associates, Inc.). Each chamber contained an active and an inactive lever, a food dispenser, the CS (light) over each lever, and a house light.

\section{Self-administration training}

Cocaine self-administration training began 5-7 d after surgery. On the first day, the animals were moved from a light/dark cycle room (lights on at 6:00 a.m.) to a reverse light/dark cycle (lights on at 6:00 p.m.) and housed in this room for the remainder of the experiment. All self-administration studies were conducted during the same time of day. On day 1 , animals were placed in the self-administration chamber for an overnight training session on a fixed ratio (FR) 1 schedule of reinforcement. Correct lever presses resulted in cocaine infusion $(0.75 \mathrm{mg} / \mathrm{kg}$ in $0.10 \mathrm{~mL}$ over $6 \mathrm{sec}$ ) and illumination of a CS light above the lever. The CS light remained on for $6 \mathrm{sec}$, while a house light was illuminated for 20 sec during which active lever presses were counted but resulted in no further cocaine infusions. After the 20 -sec house light was extinguished, the next active lever press resulted in a cocaine infusion. Presses on the inactive lever had no consequences but were recorded. Animals were continuously given overnight sessions until they received at least 20 cocaine rewards (2-4 nights). Thereafter, subsequent sessions lasted for $2 \mathrm{~h}$ each day. Each animal was placed in the same chamber each day.

The acquisition criteria for cocaine self-administration consisted of 3 consecutive days of 10 rewards during the FR1 schedule. After FR1 maintenance was stabilized, animals were switched to an FR5 schedule of reinforcement. During the FR5 schedule, animals met a criterion of 7 consecutive days of at least 10 rewards. Additionally, the last $3 \mathrm{~d}$ of the FR5 schedule must have had less than 10\% variability in active lever pressing; otherwise, the animal was kept on this schedule until active lever pressing stabilized. After stable maintenance was obtained, subjects were moved to the extinction training phase.

\section{Extinction training}

During extinction sessions, lever pressing resulted in no infusion of cocaine or in activation of the CS light or house light. Animals underwent extinction training until active lever presses were less than $10 \%$ of the last maintenance day for three consecutive days.

\section{Reactivation and reinstatement}

Once extinction training was completed, two different reactivation protocols were used: one in which cocaine was given noncontingently and animals remained in the self-administration chamber for 15 min (Experiment 3), and a second in which animals were allowed to self-administer cocaine for the standard 2-h session (Experiment 4). For both reactivation protocols, animals underwent an FR1 schedule for 2 consecutive days. On these reactivation days, animals received an injection of saline or MK$801(0.05 \mathrm{mg} / \mathrm{kg}$ or $0.20 \mathrm{mg} / \mathrm{kg}$, i.p.) $30 \mathrm{~min}$ prior to the session. After $30 \mathrm{~min}$, rats received a cocaine priming injection $(10 \mathrm{mg} /$ $\mathrm{kg}$, i.p.) before being placed into the operant chambers, and the CS was noncontingently presented one time upon entry into the chamber. During 15-min reactivation sessions, active lever pressing resulted in CS presentation and illumination of the house light but no cocaine infusion. Two-hour reactivation sessions were similar to maintenance training; thus, active lever pressing resulted in both CS presentation, the house light, and cocaine infusion $(0.75 \mathrm{mg} / \mathrm{kg})$. One day after the second reactivation session, animals underwent cocaine reinstatement. Reinstatement consisted of a cocaine priming injection $(10 \mathrm{mg} / \mathrm{kg}$, i.p.) immediately prior to placement in the self-administration chamber. All reinstatement sessions were 2-h FR1 sessions with no cocaine infusions; however, all other cues were present, including the sound of the infusion pump, the CS, and the house light.

For the self-administration studies, we chose to conduct reactivation sessions in the presence of all drug-related stimuli possible, including contextual, discrete, and interoceptive stimuli from the cocaine, to fully reactivate the memory, since we had previously found that rats required both the context of the CPP chamber and the cocaine itself to successfully disrupt reconsolidation of the cocaine-associated memory (Brown et al. 2007). On the reinstatement day, rats again received all stimuli associated with the maintenance phase of drug-taking behavior, including the discrete drug cue, since we wished to examine the impact of cocaine combined with the cocaine-associated light cue.

\section{Statistical analysis}

All data were analyzed with StatView software for Macintosh. For analysis of CPP behavior, the difference between test day and initial preference day is reported (preference score) and the number of photocell beam interruptions (to assess general activity levels) is reported. For analysis of self-administration data, lever pressing on the active lever and inactive lever are reported. Oneway analysis of variance (ANOVA) with a repeated measure over days was conducted for each treatment group to determine the effect of day of testing, followed by a Fischer's least significant difference test in the case of a significant day effect. To determine the effect of MK-801 on behavior, each dose of MK-801 was compared with Saline controls using a two-way ANOVA with a repeated measure over days followed by a Fischer's least significant difference test in the case of a significant interaction $(P<0.05)$. Data examining behavior across treatments on a single day (locomotor behavior on the reinstatement day (Figs. 1B and 2B) were assessed using a Student's two-tailed $t$-test. All group sizes are reported in the figure legends.

\section{Acknowledgments}

The authors thank Jenny Baylon for assistance with the study. This work was supported by Public Health Service grant DA 023729.

\section{References}

Alberini, C.M. 2005. Mechanisms of memory stabilization: Are consolidation and reconsolidation similar or distinct processes? Trends Neurosci. 28: 51-56.

Bardo, M.T., Valone, J.M., and Bevins, R.A. 1999. Locomotion and conditioned place preference produced by acute intravenous amphetamine: Role of dopamine receptors and individual differences in amphetamine self-administration. Psychopharmacology 143: $39-46$.

Ben Mamou, C., Gamache, K., and Nader, K. 2006. NMDA receptors are critical for unleashing consolidated auditory fear memories. Nat. Neurosci. 9: 1237-1239.

Bernardi, R.E., Lattal, K.M., and Berger, S.P. 2006. Postretrieval propranolol disrupts a cocaine conditioned place preference. Neuroreport 17: 1443-1447.

Bouton, M.E. 2004. Context and behavioral processes in extinction. Learn. Mem. 11: 485-494.

Brown, T.E., Forquer, M.R., Cocking, D.L., Jansen, H.T., Harding, J.W., and Sorg, B.A. 2007. Role of matrix metalloproteinases in the acquisition and reconsolidation of cocaine-induced conditioned place preference. Learn. Mem. 14: 214-223.

Brunet, A., Orr, S.P., Tremblay, J., Robertson, K., Nader, K., and Pitman, R.K. 2008. Effect of post-retrieval propranolol on psychophysiologic responding during subsequent script-driven traumatic imagery in post-traumatic stress disorder. J. Psychiatr. Res. 42: 503-506.

Debiec, J. and LeDoux, J.E. 2004. Disruption of reconsolidation but not consolidation of auditory fear conditioning by noradrenergic blockade in the amygdala. Neuroscience 129: 267-272. 
Debiec, J., LeDoux, J.E., and Nader, K. 2002. Cellular and systems reconsolidation in the hippocampus. Neuron 36: 527-538.

De Vries, T.J., Schoffelmeer, A.N., Binnekade, R., Mulder, A.H., and Vanderschuren, L.J. 1998. MK-801 reinstates drug-seeking behaviour in cocaine-trained rats. Neuroreport 9: 637-640.

de Wit, H. and Stewart, J. 1981. Reinstatement of cocaine-reinforced responding in the rat. Psychopharmacology 75: 134-143.

Edwards, S., Whisler, K.N., Fuller, D.C., Orsulak, P.J., and Self, D.W. 2007. Addiction-related alterations in D1 and D2 dopamine receptor behavioral responses following chronic cocaine self-administration. Neuropsychopharmacology 32: 354-366.

Epstein, D.H., Preston, K.L., Stewart, J., and Shaham, Y. 2006. Toward a model of drug relapse: An assessment of the validity of the reinstatement procedure. Psychopharmacology 189: 1-16.

Frankland, P.W., Ding, H.K., Takahashi, E., Suzuki, A., Kida, S., and Silva, A.J. 2006. Stability of recent and remote contextual fear memory. Learn. Mem. 13: 451-457.

Fricks-Gleason, A.N. and Marshall, J.F. 2008. Post-retrieval $\beta$-adrenergic receptor blockade: Effects on extinction and reconsolidation of cocaine-cue memories. Learn. Mem. 15: 643-648.

Hernandez, P.J. and Kelley, A.E. 2004. Long-term memory for instrumental responses does not undergo protein synthesis-dependent reconsolidation upon retrieval. Learn. Mem. 11: 748-754.

Jaffe, J.H., Cascella, N.G., Kumor, K.M., and Sherer, M.A. 1989. Cocaine-induced cocaine craving. Psychopharmacology 97: 59-64.

Kalivas, P.W. and McFarland, K. 2003. Brain circuitry and the reinstatement of cocaine-seeking behavior. Psychopharmacology 168: 44-56.

Kelamangalath, L., Swant, J., Stramiello, M., and Wagner, J.J. 2007. The effects of extinction training in reducing the reinstatement of drug-seeking behavior: Involvement of NMDA receptors. Behav. Brain Res. 185: 119-128.

Kelley, J.B., Anderson, K.L., and Itzhak, Y. 2007. Long-term memory of cocaine-associated context: Disruption and reinstatement. Neuroreport 18: 777-780.

Lee, J.L. and Everitt, B.J. 2008. Appetitive memory reconsolidation depends upon NMDA receptor-mediated neurotransmission. Neurobiol. Learn. Mem. 90: 147-154.

Lee, J.L., Di Ciano, P., Thomas, K.L., and Everitt, B.J. 2005. Disrupting reconsolidation of drug memories reduces cocaine-seeking behavior. Neuron 47: 795-801.

Lee, J.L., Milton, A.L., and Everitt, B.J. 2006a. Cue-induced cocaine seeking and relapse are reduced by disruption of drug memory reconsolidation. J. Neurosci. 26: 5881-5887.

Lee, J.L., Milton, A.L., and Everitt, B.J. 2006b. Reconsolidation and extinction of conditioned fear: Inhibition and potentiation. $J$. Neurosci. 26: 10051-10056.

Leri, F., Zhou, Y., Goddard, B., Cummins, E., and Kreek, M.J. 2006 Effects of high-dose methadone maintenance on cocaine place conditioning, cocaine self-administration, and $\mu$-opioid receptor mRNA expression in the rat brain. Neuropsychopharmacology 31: $1462-1474$.

Litvin, O.O. and Anokhin, K.V. 2000. Mechanisms of memory reorganization during retrieval of acquired behavioral experience in chicks: The effects of protein synthesis inhibition in the brain. Neurosci. Behav. Physiol. 30: 671-678.

Ludwig, A.M., Wikler, A., and Stark, L.H. 1974. The first drink: Psychobiological aspects of craving. Arch. Gen. Psychiatry 30: 539-547.

McFarland, K. and Ettenberg, A. 1997. Reinstatement of drug-seeking behavior produced by heroin-predictive environmental stimuli. Psychopharmacology 131: 86-92.

McFarland, K. and Kalivas, P.W. 2001. The circuitry mediating cocaine-induced reinstatement of drug-seeking behavior. J. Neurosci. 21: 8655-8663.

Milekic, M.H. and Alberini, C.M. 2002. Temporally graded requirement for protein synthesis following memory reactivation. Neuron 36: 521-525.

Milekic, M.H., Brown, S.D., Castellini, C., and Alberini, C.M. 2006. Persistent disruption of an established morphine conditioned place preference. J. Neurosci. 26: 3010-3020.

Miller, C.A. and Marshall, J.F. 2005. Molecular substrates for retrieval and reconsolidation of cocaine-associated contextual memory. Neuron 47: 873-884.

Milton, A.L., Lee, J.L., and Everitt, B.J. 2008a. Reconsolidation of appetitive memories for both natural and drug reinforcement is dependent on $\beta$-adrenergic receptors. Learn. Mem. 15: 88-92.

Milton, A.L., Lee, J.L.C., Butler, V.J., Gardner, R., and Everitt, B.J. 2008b. Intra-amygdala and systemic antagonism of NMDA receptors prevents the reconsolidation of drug-associated memory and impairs subsequently both novel and previously acquired drug-seeking behaviors. J. Neurosci. 28: 8230-8237.

Misanin, J.R., Miller, R.R., and Lewis, D.J. 1968. Retrograde amnesia produced by electroconvulsive shock after reactivation of a consolidated memory trace. Science 160: 554-555.

Mueller, D. and Stewart, J. 2000. Cocaine-induced conditioned place preference: Reinstatement by priming injections of cocaine after extinction. Behav. Brain Res. 115: 39-47.

Nader, K., Schafe, G.E., and Le Doux, J.E. 2000a. Fear memories require protein synthesis in the amygdala for reconsolidation after retrieval. Nature 406: 722-726.

Nader, K., Schafe, G.E., and LeDoux, J.E. 2000b. The labile nature of consolidation theory. Nat. Rev. Neurosci. 1: 216-219.

Panos, J.J., Rademacher, D.J., Renner, S.L., and Steinpreis, R.E. 1999. The rewarding properties of NMDA and MK-801 (dizocilpine) as indexed by the conditioned place preference paradigm. Pharmacol. Biochem. Behav. 64: 591-595.

Parker, L.A. and McDonald, R.V. 2000. Reinstatement of both a conditioned place preference and a conditioned place aversion with drug primes. Pharmacol. Biochem. Behav. 66: 559-561.

Pierce, R.C., Meil, W.M., and Kalivas, P.W. 1997. The NMDA antagonist, dizocilpine, enhances cocaine reinforcement without influencing mesoaccumbens dopamine transmission. Psychopharmacology 133: 188-195.

Przybyslawski, J. and Sara, S.J. 1997. Reconsolidation of memory after its reactivation. Behav. Brain Res. 84: 241-246.

Riccio, D.C., Millin, P.M., and Bogart, A.R. 2006. Reconsolidation: A brief history, a retrieval view, and some recent issues. Learn. Mem. 13: $536-544$.

Robinson, M.J. and Franklin, K.B. 2007a. Central but not peripheral beta-adrenergic antagonism blocks reconsolidation for a morphine place preference. Behav. Brain Res. 182: 129-134.

Robinson, M.J. and Franklin, K.B. 2007b. Effects of anisomycin on consolidation and reconsolidation of a morphine-conditioned place preference. Behav. Brain Res. 178: 146-153.

Sadler, R., Herzig, V., and Schmidt, W.J. 2007. Repeated treatment with the NMDA antagonist MK-801 disrupts reconsolidation of memory for amphetamine-conditioned place preference. Behav. Pharmacol. 18: $699-703$.

Sanchez, C.J. and Sorg, B.A. 2001. Conditioned fear stimuli reinstate cocaine-induced conditioned place preference. Brain Res. 908: 86-92.

Sara, S.J. 2000. Retrieval and reconsolidation: Toward a neurobiology of remembering. Learn. Mem. 7: 73-84.

Steinpreis, R.E., Kramer, M.A., Mix, K.S., and Piwowarczyk, M.C. 1995 The effects of MK801 on place conditioning. Neurosci. Res. 22: 427-430.

Stewart, R.B. and Grupp, L.A. 1989. Conditioned place aversion mediated by self-administered ethanol in the rat: A consideration of blood ethanol levels. Pharmacol. Biochem. Behav. 32: 431-437.

Suzuki, A., Josselyn, S.A., Frankland, P.W., Masushige, S., Silva, A.J., and Kida, S. 2004. Memory reconsolidation and extinction have distinct temporal and biochemical signatures. J. Neurosci. 24: 4787-4795.

Valjent, E., Corbille, A.G., Bertran-Gonzalez, J., Herve, D., and Girault, J.A. 2006. Inhibition of ERK pathway or protein synthesis during reexposure to drugs of abuse erases previously learned place preference. Proc. Natl. Acad. Sci. 103: 2932-2937.

Yim, A.J., Moraes, C.R.G., Ferreira, T.L., and Oliveira, M.G.M. 2006 Protein synthesis inhibition in the basolateral amygdala following retrieval does not impair expression of morphine-associated conditioned place preference. Behav. Brain Res. 171: 162-169.

Received July 11, 2008; accepted in revised form October 7, 2008. 


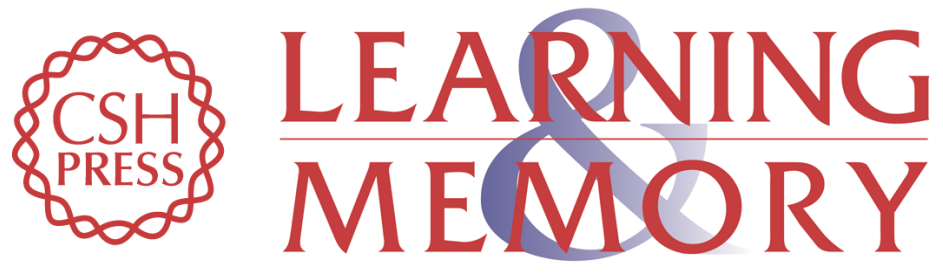

\section{The NMDA antagonist MK-801 disrupts reconsolidation of a cocaine-associated memory for conditioned place preference but not for self-administration in rats}

Travis E. Brown, Brian R. Lee and Barbara A. Sorg

Learn. Mem. 2008, 15:

Access the most recent version at doi:10.1101/lm.1152808

References This article cites 53 articles, 16 of which can be accessed free at: http://learnmem.cshlp.org/content/15/12/857.full.html\#ref-list-1

License

Email Alerting

Receive free email alerts when new articles cite this article - sign up in the box at the Service top right corner of the article or click here. 